Gyorki David (Orcid ID: 0000-0002-3165-4694)

Henderson Michael A (Orcid ID: 0000-0002-2496-8572)

Lonie Sarah (Orcid ID: 0000-0001-8109-6507)

1

\title{
MANAGEMENT OF PLEOMORPHIC DERMAL SARCOMA
}

Sarah Lonie (MBBS, BMedSci) ${ }^{1}$, Benny Yau (MBBS, BSc, $)^{1}$, Michael Henderson (MBBS, , FRACS) $)^{1,2}$, David Gyorki (MBBS, FRACS) ${ }^{1,2}$, Christopher Angel (MBBS, FRCPA) ${ }^{3}$, Angela Webb (MBBS, FRACS (Plast)) $)^{1,2}$

${ }^{1}$ Division of Cancer Surgery, Peter MacCallum Cancer Centre, Melbourne, Victoria

${ }^{2}$ Department of Surgery, University of Melbourne

${ }^{3}$ Department of Anatomical Pathology, Peter MacCallum Cancer Centre, Melbourne, Victoria

\section{Corresponding author:}

Sarah Lonie

Department of Cancer Surgery

Peter MacCallum Cancer Centre, 305 Grattan Street, Melbourne 3000, Victoria, Australia

Email: sjlonie88@gmail.com

Ph: 0419106782

\section{Presented at}

Royal Australasian College of Surgeons Annual Scientific Congress (May 2018) - poster and mini-oral.

This is the author manuscript accepted for publication and has undergone full peer review but has not been through the copyediting, typesetting, pagination and proofreading process, which may lead to differences between this version and the Version of Record. Please cite this article as doi: 10.1111/ans.15909

This article is protected by copyright. All rights reserved. 
Word count: 1715 words

\section{Abstract: 245 words}

Tables: 1

\section{Background}

Pleomorphic dermal sarcoma (PDS) is a rare, poorly defined skin neoplasm with features similar to atypical fibroxanthoma, but with adverse histopathologic characteristics indicating metastatic potential such as tumour necrosis, invasion beyond superficial subcutis or vascular and/or perineural infiltration. Optimal treatment for PDS is uncertain and reported outcomes vary due to the rarity of this diagnosis and uncertainty over histopathological categorisation. The aim of this study was to review the clinical and histopathological features of PDS in a single Australian centre.

\section{Method}

A retrospective review of all patients managed at Peter MacCallum Cancer Centre with PDS between 2003-2017 was performed by a search of electronic records and histories reviewed.

\section{Results}

Twenty-seven patients were identified, mostly elderly males (85.2\%, mean age 79.8 years). Lesions were seen most commonly on the head and neck region (96.3\%); predominantly on the scalp (63\%). Mean tumour radial surgical excision margin was $12.8 \mathrm{~mm}$. 18 patients $(66.7 \%)$ underwent radiotherapy, 13 adjuvant, 3 neo-adjuvant, and 2 with palliative intent. After median follow-up of 46.4 months, two patients had recurrence (7.4\%); both had inadequate deep

This article is protected by copyright. All rights reserved. 
margins at first excision. There were three all-cause deaths in the cohort. There was one disease specific mortality with metastatic PDS disease at time of initial presentation.

\section{Conclusion}

PDS is a rare cutaneous malignancy most commonly found in the head and neck region in elderly men, which is best managed with adequate surgical excision. The role of radiotherapy is undefined and an area for future investigation.

Keywords: skin neoplasm, sarcoma, skin surgery, treatment, surgical oncology

\section{Background}

Pleomorphic dermal sarcoma (PDS), formerly referred to as malignant fibrous histiocytoma (MFH) of skin, was recently recognised by the 2013 World Health Organisation (WHO) classification of soft tissue tumours as a separate entity ${ }^{1-3}$. MFH was previously described as a sarcoma with pleomorphic phenotype and an unknown line of differentiation. With advances in immunohistochemistry, many of these tumours can now be classified and the remaining truly undifferentiated pleomorphic sarcomas have been more accurately categorised. PDS is a rare, poorly-defined dermal neoplasm most commonly affecting sun-exposed skin of elderly males ${ }^{4}$. It is the cutaneous variant of undifferentiated pleomorphic sarcoma. The clinical presentation of PDS is usually of a rapid growing, exophytic, ulcerated and bleeding tumour ${ }^{4}$. The morphological features of PDS are similar to atypical fibroxanthoma (AFX) ${ }^{4}$. Histologically, PDS and AFX comprise sheets of pleomorphic, bizarre-appearing epithelioid and spindle cells with abundant cytoplasm, hyperchromatic nuclei and multiple nucleoli ${ }^{5}$. Multinucleated giant cells are often seen and numerous atypical mitoses are often present. Other tumours such as melanoma, poorly differentiated carcinoma and other soft tissue neoplasms need to be excluded (largely via immunohistochemistry). The key defining factors of PDS, to differentiate it 
from AFX, include tumour necrosis, invasion beyond the superficial subcutis or vascular and/or perineural infiltration, indicating possible metastatic potential ${ }^{4}$.

The recent changes in nomenclature help create homogeneous diagnostic criteria but have not removed clinical uncertainty regarding management and expected outcomes of PDS. The mainstay of treatment for PDS is surgical excision however optimal margins are poorly defined and there is little evidence regarding the role of adjuvant radiotherapy.

The aim of this report was to document our experience with the management of pleomorphic dermal sarcoma, through a retrospective review of all cases at a tertiary referral cancer centre. To date, there are two other series reporting on pleomorphic sarcoma in the literature with histopathology and immunohistochemistry documentation; however few studies report on ideal management and outcomes ${ }^{6,7}$.

\section{Method}

This retrospective study identified all patients managed at Peter MacCallum Cancer Centre, Melbourne, with PDS between 2003-2017. The pathology department database and hospital electronic records were searched to identify all patients with a diagnosis of PDS. Patient histories were reviewed for demographic data, histology and immunohistochemistry, management (surgical, radiation or chemotherapy) and outcome data. Approval was obtained from Peter MacCallum Cancer Centre Human Research and Ethics Committee (HREC/17/69R). 
The standard of care at Peter MacCallum was for patients to have a wide local excision of PDS with a minimum 10mm margin. Radiotherapy was usually recommended after a multidisciplinary conference.

\section{Statistical analysis}

Analyses were performed using STATA (version 12, Stata Corp LP, College Station, TX) software. ANOVA [Analysis of Variance] and chi-square tests were used to compare groups. A value of $p<0.05$ was considered to indicate significant differences between groups.

\section{Results}

\section{DEMOGRAPHICS}

Twenty-seven patients met the inclusion criteria. Patients were mean age 79.8 , with a majority being male (85.2\%). There was a history of chronic sun damage in $59.3 \%$ of patients. Five (18.5\%) patients had a degree of immune suppression. Nearly all tumours were located on the head and neck region with the majority on the scalp (63\%). Mean depth of invasion was $7.3 \mathrm{~mm}$ (interquartile range $(\mathrm{IQR})=5 \mathrm{~mm})$. On immunohistochemistry, $81.5 \%$ stained positive for $\mathrm{CD} 10$ (81.5\%), none had staining of S100 protein, CD34 or keratins. Mitoses were reported as numerous in $35 \%$ and frequent in $33.3 \%$.

Twenty-six patients were managed by surgery alone (96.3\%). Mean radial surgical margin was 12.8mm (IQR=15mm). There was one patient who did not have surgical resection as the patient presented with metastatic PDS.

This article is protected by copyright. All rights reserved. 
Eighteen (66.7\%) patients received radiotherapy; 16 with curative intent and 2 with palliative intent. Palliative radiotherapy was given to a patient with bone metastases at the time of diagnosis and also to a patient with nodal metastases after surgical resection of the primary lesion. Patients who underwent radiotherapy tended to be younger (mean age 76.7 vs. 84.4, $p=0.1$ ) with no significant differences in terms of tumour depth or location compared to those who received no radiation (Table 1).

Median patient follow-up was 46.4 months. There were two cases of recurrence and three cases of all-cause mortality, but only one disease-specific mortality; this patient had metastatic disease at the time of primary diagnosis.

The first patient with recurrent PDS was an 89-year old man previously managed at another institution, who did not undergo wide local excision after an incomplete excision of a scalp PDS. A satellite nodule was excised 16 months following this first excision with a $0.3 \mathrm{~mm}$ deep margin and then radiotherapy was delivered to this area. Further satellite nodules of PDS developed around the scalp during the radiation course. Palliative radiotherapy was then delivered to these areas for symptom control. He died of an unrelated cause 15 months post-radiation therapy, having had no further symptomatic recurrence.

The second patient was a 74-year old man, with scalp PDS who had two primary excisions to gain clearance (first involving deep margin, second $1 \mathrm{~mm}$ clear from deep margin and outer table of skull bone burred). Despite adjuvant radiotherapy he developed dermal metastases and subsequently parotid node metastases 8 months post-operatively. He underwent resection of symptomatic lesions, superficial parotidectomy and chemotherapy with doxorubicin. He remains alive with disease now 22 months following first excision.

This article is protected by copyright. All rights reserved. 


\section{Discussion}

This study; a retrospective review of PDS, is one of the largest series reporting on management outcomes of this rare tumour.

There are two other series published on PDS, both with a higher recurrence rate, than our cohort. The previous literature does not clearly report on adjuvant radiation treatment for this tumour. Miller et al present their series of 32 patients, with local recurrence in 8 (28\% of patients), of whom seven tumours were previously incompletely excised ${ }^{6}$. Distant disease developed in $9 \%{ }^{6}$. They also noted a high degree of intercurrent illness such as leukaemia, lymphoma and other malignancies in nearly half and $41 \%$ died of unrelated causes. In their group, PDS occurred in head and neck region in all cases but one on the forearm, in mostly males (7:1 male to female). Of the three patients who developed metastases, two had multiple cutaneous deposits and one nodal metastasis. The nodal metastatic disease was managed with chemotherapy, which achieved disease-free survival at 79 months follow-up. One patient died after a second recurrence in the setting of advanced non-Hodgkin lymphoma.

Tardio et al. present their series of 15 patients with a local recurrence rate of $20 \%$ and distant metastasis rate of $20 \%$ over mean follow up of 33 months $^{7}$. All 15 cases occurred in the head and neck region and patients had a mean age of 81 years. All three patients with local recurrence had incomplete primary excision margins. Three other patients developed nodal or lung metastases (one with incomplete primary excision). Of the three patients with pulmonary metastases, one had received adjuvant radiation to the tumour bed and the other went on to receive radiation following lymphadenectomy for nodal spread. All patients who developed 
metastases died from disease between 12-33 months following diagnosis. The two cases who developed metastases despite complete primary surgical resection were the largest tumours in the series (45mm and $70 \mathrm{~mm})$. Unfortunately, it is uncertain which patients received adjuvant radiation in the course of their treatment.

Our study population supports previous findings that PDS is a malignancy most likely to occur on the scalp region in elderly males. There is an association with chronic sun damage and immune-suppression. As a deeply invasive malignancy, wide clear surgical margins appear key to gaining local control, with an average wide-excision radial margin of $12.8 \mathrm{~mm}$. In our cohort, two cases clinically recurred (7.4\%), one with previous incomplete margins and one who had received radiotherapy who then also developed nodal metastasis. If the case with previous incomplete margins is considered residual disease, the recurrence rate in our series is $3.7 \%$. This is considerably lower than reports from other series which consider cases with incomplete primary excision developing visible clinical disease to be recurrence rather than residual disease.

One other case in the current series developed nodal disease prior to any surgical intervention. Due to his comorbidities, he was not deemed fit for surgery and was the only disease specific mortality in this series. This highlights that PDS has the potential to be aggressive and metastasise if not controlled locally with timely surgical wide excision.

Radiotherapy should be considered judiciously as patients with PDS are often elderly with a high degree of intercurrent illness. We found $11.1 \%$ of patients died of unrelated causes in the follow-up period. Overall, in management of PDS, adequate wide-excision should be considered 
foremost to obtain local disease control. The role of radiation as adjuvant treatment for may be best limited to patients with high-risk features such as perineural or lymphovascular invasion. Future longitudinal and prospective investigation into this relationship must be performed to further delineate the utility of adjuvant radiation therapy in the management of PDS.

Chemotherapy is also an area that requires further investigation in relation to PDS management. It has been used as a salvage option once nodal metastases have developed. Miller et $\mathrm{al}^{6}$ describe their patient with nodal spread managed with chemotherapy achieving disease-free survival 79 months after follow-up. In our cohort, a patient with parotid nodal metastases remains alive with disease 22 months following first excision and is receiving adjuvant chemotherapy.

\section{Conclusion}

PDS is a rare cutaneous malignancy most commonly found in the head and neck region in elderly men, which is well controlled with appropriate surgical excision. In our series of 27 patients, once wide surgical excision was obtained, only one had true local recurrence (3.7\%). The role of adjuvant radiotherapy in the setting of complete surgical excision remains unclear at this time and warrants further research. Radiotherapy and chemotherapy have a role to play in recurrent and metastatic cases where surgical excision is not possible.

\section{Disclosure statement}

No conflicts of interest or financial support to declare

This article is protected by copyright. All rights reserved. 


\section{References}

[1] McCalmont TH. Correction and clarification regarding AFX and pleomorphic dermal sarcoma. Journal of cutaneous pathology. 2012; 39:8.

[2] McCalmont TH. AFX: what we now know. Journal of cutaneous pathology. 2011; 38:853-6.

[3] Calonje B, Komminoth. WHO Classification of tumours of soft tissue and bone: International Agency for Research on Cancer, 2013.

[4] Brenn T. Pleomorphic dermal neoplasms: a review. Advances in anatomic pathology. 2014; 21:108-30.

[5] Soleymani T, Tyler Hollmig S. Conception and Management of a Poorly Understood Spectrum of Dermatologic Neoplasms: Atypical Fibroxanthoma, Pleomorphic Dermal Sarcoma, and Undifferentiated Pleomorphic Sarcoma. Current treatment options in oncology. $2017 ; 18: 50$.

[6] Miller K, Goodlad JR, Brenn T. Pleomorphic dermal sarcoma: adverse histologic features predict aggressive behavior and allow distinction from atypical fibroxanthoma. The American journal of surgical pathology. 2012; 36:1317-26.

[7] Tardio JC, Pinedo F, Aramburu JA, et al. Pleomorphic dermal sarcoma: a more aggressive neoplasm than previously estimated. Journal of cutaneous pathology. 2016; 43:101-12.

Figure legend

This article is protected by copyright. All rights reserved. 
Table 1: Comparison of irradiated and non-irradiated patients

This article is protected by copyright. All rights reserved. 
Table 1: Comparison of irradiated and non-irradiated patients

\begin{tabular}{|llll|}
\hline Demographics & Patients who received & No radiation (n=9) & Significance \\
& radiation $(\mathbf{n = 1 8})$ & & 0.1 \\
\hline Age (mean, years) & 76.7 & 84.4 & 0.06 \\
\hline Gender (male) & $17(94.4 \%)$ & $6(66.7 \%)$ & 0.48 \\
\hline Immune suppressed & $4(22.2 \%)$ & $1(11.1 \%)$ & \\
\hline Location & Head and neck $=18$ & Head and neck $=8$ & \\
& - Scalp $=12(66.7 \%)$ & - Scalp $=5(55.6 \%)$ & \\
& - Face $=5(27.8 \%)$ & - Face $=2(22.2 \%)$ & \\
& - Ear $=1(5.6 \%)$ & - Ear $=1(11.1 \%)$ & 0.10 \\
\hline Recurrence & $1(5.6 \%)$ & Torso $=1(11.1 \%)$ & \\
\hline
\end{tabular}

This article is protected by copyright. All rights reserved. 
Table 1: Comparison of irradiated and non-irradiated patients

\begin{tabular}{|llll|}
\hline Demographics & Patients who received & No radiation (n=9) & Significance \\
\hline Age (mean, years) & 76.7 & & \\
\hline Gender (male) & $17(94.4 \%)$ & 64.4 & 0.1 \\
\hline Immune suppressed & $4(22.2 \%)$ & $1(11.1 \%)$ & 0.48 \\
\hline Location & Head and neck $=18$ & Head and neck $=8$ & \\
& - Scalp $=12(66.7 \%)$ & - Scalp $=5(55.6 \%)$ & \\
& - Face $=5(27.8 \%)$ & - Face $=2(22.2 \%)$ & \\
& - Ear $=1(5.6 \%)$ & - Ear $=1(11.1 \%)$ & \\
\hline Recurrence & $1(5.6 \%)$ & Torso $=1(11.1 \%)$ & 0.10 \\
\hline
\end{tabular}

This article is protected by copyright. All rights reserved. 


\section{University Library}

\section{- M M N E R VA A gateway to Melbourne's research publications}

Minerva Access is the Institutional Repository of The University of Melbourne

Author/s:

Lonie, S;Yau, B;Henderson, M;Gyorki, D;Angel, C;Webb, A

Title:

Management of pleomorphic dermal sarcoma

Date:

2020-04-27

Citation:

Lonie, S., Yau, B., Henderson, M., Gyorki, D., Angel, C. \& Webb, A. (2020). Management of pleomorphic dermal sarcoma. ANZ JOURNAL OF SURGERY, 90 (11), pp.2322-2324. https:// doi.org/10.1111/ans. 15909.

Persistent Link:

http://hdl.handle.net/11343/275686 